\title{
IMPROVEMENT OF FRACTIONAL HERMITE-HADAMARD TYPE INEQUALITY FOR CONVEX FUNCTIONS
}

\author{
MEHMET KUNT, IMDAT IŞCAN, SERCAN TURHAN, AND DÜNYA KARAPINAR
}

Received 02 November, 2017

\begin{abstract}
In this paper, it is proved that fractional Hermite-Hadamard inequality and fractional Hermite-Hadamard-Fejér inequality are just results of Hermite-Hadamard-Fejér inequality. After this, a new fractional Hermite-Hadamard inequality which is not a result of Hermite-HadamardFejér inequality and better than given in [9] by Sarıkaya et al. is obtained. Also, a new equality is proved and some new fractional midpoint type inequalities are given. Our results generalizes the results given in [5] by Kirmaci.
\end{abstract}

2010 Mathematics Subject Classification: 26A51; 26A33; 26D10

Keywords: convex functions, Hermite-Hadamard inequalities, Riemann-Liouville fractional integrals, midpoint type inequalities

\section{INTRODUCTION}

Let $f: I \subseteq \mathbb{R} \rightarrow \mathbb{R}$ be a convex function defined on the interval $I$ of real numbers and $a, b \in I$ with $a<b$. The inequality

$$
f\left(\frac{a+b}{2}\right) \leq \frac{1}{b-a} \int_{a}^{b} f(x) d x \leq \frac{f(a)+f(b)}{2}
$$

is well known in the literature as Hermite-Hadamard's inequality $[2,3]$.

The most well-known inequalities related to the integral mean of a convex function $f$ are the Hermite Hadamard inequality or its weighted versions, the so-called Hermite-Hadamard-Fejér inequality.

In [1], Fejér established the following Fejér inequality which is the weighted generalization of Hermite-Hadamard inequality (1.1):

Theorem 1. Let $f:[a, b] \rightarrow \mathbb{R}$ be convex function. Then, the inequality

$$
f\left(\frac{a+b}{2}\right) \int_{a}^{b} g(x) d x \leq \int_{a}^{b} f(x) g(x) d x \leq \frac{f(a)+f(b)}{2} \int_{a}^{b} g(x) d x
$$

holds, where $g:[a, b] \rightarrow \mathbb{R}$ is nonnegative, integrable and symmetric to $\frac{a+b}{2}$ (i.e. $g(x)=g(a+b-x)$ for all $x \in[a, b])$. 
In [5], Kurmaci used the following equality to obtain midpoint type inequalities and some applications:

Lemma 1. Let $a, b \in I$ with $a<b$ and $f: I^{\circ} \rightarrow \mathbb{R}$ is a differentiable mapping $\left(I^{\circ}\right.$ the interior of $\left.I\right)$. If $f^{\prime} \in L[a, b]$, then we have

$$
\begin{aligned}
& \frac{1}{b-a} \int_{a}^{b} f(u) d u-f\left(\frac{a+b}{2}\right) \\
& =(b-a) \int_{0}^{1 / 2} t f^{\prime}(t a+(1-t) b) d t+\int_{1 / 2}^{1}(t-1) f^{\prime}(t a+(1-t) b) d t .
\end{aligned}
$$

Following definitions of the left and right side Riemann-Liouville fractional integrals are well known in the literature.

Definition 1. Let $a, b \in \mathbb{R}$ with $a<b$ and $f \in L[a, b]$. The left and right RiemannLiouville fractional integrals $R_{a+}^{\theta} f$ and $R_{b-}^{\theta} f$ of order $\theta>0$ are defined by

$$
R_{a+}^{\theta} f(x)=\frac{1}{\Gamma(\theta)} \int_{a}^{x}(x-t)^{\theta-1} f(t) d t, x>a
$$

and

$$
R_{b-}^{\theta} f(x)=\frac{1}{\Gamma(\theta)} \int_{x}^{b}(t-x)^{\theta-1} f(t) d t, x<b
$$

respectively, where $\Gamma(\theta)$ is the Gamma function defined by $\Gamma(\theta)=\int_{0}^{\infty} e^{-t} t^{\theta-1} d t$ (see [6, page 69] and [10, page 4]).

In [9], Sarıkaya et al. proved the following fractional Hermite-Hadamard type inequality:

Theorem 2. Let $f:[a, b] \rightarrow \mathbb{R}$ be a positive function with $0 \leq a<b$ and $f \in$ $L[a, b]$. If $f$ is a convex function on $[a, b]$, then the following inequalities for fractional integrals holds:

$$
f\left(\frac{a+b}{2}\right) \leq \frac{\Gamma(\theta+1)}{2(b-a)^{\theta}}\left[R_{a+}^{\theta} f(b)+R_{b-}^{\theta} f(a)\right] \leq \frac{f(a)+f(b)}{2}
$$

with $\theta>0$.

Remark 1. In Theorem 2, it is not necessary supposing that $f$ be a positive function and $a, b$ are positive real numbers. From the Definition 1 , it is clear that $a, b$ are any real numbers such as $a<b$.

In [4], İşcan proved the following fractional Hermite-Hadamard-Fejér type inequality: 
Theorem 3. Let $f:[a, b] \rightarrow \mathbb{R}$ be a convex function with $a<b$ and $f \in L[a, b]$. If $g:[a, b] \rightarrow \mathbb{R}$ is nonnegative, integrable and symmetric to $\frac{a+b}{2}$, then the following inequality for fractional integrals holds:

$$
\begin{aligned}
f\left(\frac{a+b}{2}\right)\left[R_{a+}^{\theta} g(b)+R_{b-}^{\theta} g(a)\right] & \leq\left[R_{a+}^{\theta}(f g)(b)+R_{b-}^{\theta}(f g)(a)\right] \\
& \leq \frac{f(a)+f(b)}{2}\left[R_{a+}^{\theta} g(b)+R_{b-}^{\theta} g(a)\right]
\end{aligned}
$$

with $\theta>0$.

In [7], Kunt et al. proved the following left Riemann-Liouville fractional HermiteHadamard type inequality and next equality:

Theorem 4. Let $a, b \in \mathbb{R}$ with $a<b$ and $f:[a, b] \rightarrow \mathbb{R}$ be a convex function. If $f \in L[a, b]$, then the following inequality for the left Riemann-Liouville fractional integral holds:

$$
f\left(\frac{\theta a+b}{\theta+1}\right) \leq \frac{\Gamma(\theta+1)}{(b-a)^{\theta}} R_{a+}^{\theta} f(b) \leq \frac{\theta f(a)+f(b)}{\theta+1}
$$

with $\theta>0$.

Lemma 2. Let $a, b \in \mathbb{R}$ with $a<b$ and $f:[a, b] \rightarrow \mathbb{R}$ be a differentiable function on $(a, b)$. If $f^{\prime} \in L[a, b]$, then the following equality for the left Riemann-Liouville fractional integrals holds:

$$
\begin{aligned}
\frac{\Gamma(\theta+1)}{(b-a)^{\theta}} R_{a+}^{\theta} f(b) & -f\left(\frac{\theta a+b}{\theta+1}\right) \\
& =(b-a)\left[\begin{array}{c}
\int_{0}^{\frac{\theta}{\theta+1}} t^{\theta} f^{\prime}(t a+(1-t) b) d t \\
+\int_{\frac{\theta}{\theta+1}}^{1}\left(t^{\theta}-1\right) f^{\prime}(t a+(1-t) b) d t
\end{array}\right]
\end{aligned}
$$

with $\theta>0$.

In [8], Kunt et al. proved the following right Riemann-Liouville fractional HermiteHadamard type inequality and next equality:

Theorem 5. Let $a, b \in \mathbb{R}$ with $a<b$ and $f:[a, b] \rightarrow \mathbb{R}$ be a convex function. If $f \in L[a, b]$, then the following inequality for the right Riemann-Liouville fractional integral holds:

$$
f\left(\frac{a+\theta b}{\theta+1}\right) \leq \frac{\Gamma(\theta+1)}{(b-a)^{\theta}} R_{b-}^{\theta} f(a) \leq \frac{f(a)+\theta f(b)}{\theta+1}
$$

with $\theta>0$. 
Lemma 3. Let $a, b \in \mathbb{R}$ with $a<b$ and $f:[a, b] \rightarrow \mathbb{R}$ be a differentiable function on $(a, b)$. If $f^{\prime} \in L[a, b]$, then the following equality for the right Riemann-Liouville fractional integrals holds:

$$
\begin{aligned}
\frac{\Gamma(\theta+1)}{(b-a)^{\theta}} R_{b-}^{\theta} f(a) & -f\left(\frac{a+\theta b}{\theta+1}\right) \\
& =(b-a)\left[\begin{array}{c}
\int_{0}^{\frac{\theta}{\theta+1}}-t^{\theta} f^{\prime}(t b+(1-t) a) d t \\
+\int_{\frac{\theta}{\theta+1}}^{1}\left(1-t^{\theta}\right) f^{\prime}(t b+(1-t) a) d t
\end{array}\right]
\end{aligned}
$$

with $\theta>0$.

In our studies we noticed that fractional Hermite-Hadamard type inequality given in Theorem 2 and fractional Hermite-Hadamard-Fejér type inequality given in Theorem 3 are just result of Hermite-Hadamard-Fejér inequality (given in Theorem 1), with a special selection of the weighted function. This show how strong the HermiteHadamard-Fejér inequality is. However, we will prove new fractional Hermite-Hadamard type inequality which is not a result of Theorem 1. Also, we will have new fractional midpoint type inequalities.

\section{Results of HeRmite-HADAMARD-FEJÉR INEQUALITY}

Proposition 1. Theorem 2 is a result of Theorem 1.

Proof. In Theorem 1, let we choose $g(x)=(x-a)^{\theta-1}+(b-x)^{\theta-1}$ for $\theta>0$, $a, b \in \mathbb{R}$ and $g:[a, b] \rightarrow \mathbb{R}$ (It is clear $g(x)$ nonnegative, integrable and symmetric to $\left.\frac{a+b}{2}\right)$. Computing the following integrals, we have

$$
\begin{gathered}
\int_{a}^{b} g(x) d x=\int_{a}^{b}(x-a)^{\theta-1}+(b-x)^{\theta-1} d x=\frac{2(b-a)^{\theta}}{\theta} \\
\int_{a}^{b} f(x) g(x) d x=\int_{a}^{b}\left[(x-a)^{\theta-1}+(b-x)^{\theta-1}\right] f(x) d x \\
=\int_{a}^{b}(x-a)^{\theta-1} f(x) d x+\int_{a}^{b}(b-x)^{\theta-1} f(x) d x \\
=\Gamma(\theta)\left[R_{a+}^{\theta} f(b)+R_{b-}^{\theta} f(a)\right] .
\end{gathered}
$$

Combining (1.2), (2.1) and (2.2) we have (1.4). This completes the proof.

Proposition 2. Theorem 3 is a result of Theorem 1.

Proof. In Theorem 1, let we choose $w(x)=\left[(x-a)^{\theta-1}+(b-x)^{\theta-1}\right] g(x)$ for $\theta>0, a, b \in \mathbb{R}, g:[a, b] \rightarrow \mathbb{R}$ and $g(x)$ nonnegative, integrable and symmetric to 
$\frac{a+b}{2}$ (It is clear $w(x)$ nonnegative, integrable and symmetric to $\frac{a+b}{2}$ ). Computing the following integrals, we have

$$
\begin{gathered}
\int_{a}^{b} w(x) d x=\int_{a}^{b}\left[(x-a)^{\theta-1}+(b-x)^{\theta-1}\right] g(x) d x \\
=\int_{a}^{b}(x-a)^{\theta-1} g(x) d x+\int_{a}^{b}(b-x)^{\theta-1} g(x) d x \\
=\Gamma(\theta)\left[R_{a+g}^{\theta} g(b)+R_{b-}^{\theta} g(a)\right], \\
\int_{a}^{b} f(x) w(x) d x=\int_{a}^{b}\left[(x-a)^{\theta-1}+(b-x)^{\theta-1}\right] f(x) g(x) d x \\
=\int_{a}^{b}(x-a)^{\theta-1} f(x) g(x) d x+\int_{a}^{b}(b-x)^{\theta-1} f(x) g(x) d x \\
=\Gamma(\theta)\left[R_{a+}^{\theta}(f g)(b)+R_{b-}^{\theta}(f g)(a)\right] .
\end{gathered}
$$

Combining (1.2), (2.3) and (2.4) we have (1.5). This completes the proof.

Remark 2. Theorem 4 and Theorem 5 are not results of Theorem 1.

\section{IMPROVEMENT OF FRACTIONAL HERMite-HADAMARD TYPE INEQUALITY}

We will use Theorem 4 and Theorem 5 to have new fractional Hermite-Hadamard type inequality better than (1.4).

Theorem 6. Let $a, b \in \mathbb{R}$ with $a<b$ and $f:[a, b] \rightarrow \mathbb{R}$ be a convex function. If $f \in L[a, b]$, then the following inequality for fractional integral holds:

$$
\frac{f\left(\frac{\theta a+b}{\theta+1}\right)+f\left(\frac{a+\theta b}{\theta+1}\right)}{2} \leq \frac{\Gamma(\theta+1)}{2(b-a)^{\theta}}\left[R_{a+}^{\theta} f(b)+R_{b-}^{\theta} f(a)\right] \leq \frac{f(a)+f(b)}{2}
$$

with $\theta>0$.

Proof. If (1.6) and (1.8) gather side by side and dividing into 2, it is hold the desired result.

Remark 3. Since, $f$ is a convex function on $[a, b]$, it is clear $f\left(\frac{a+b}{2}\right) \leq$ $\frac{f\left(\frac{\theta a+b}{\theta+1}\right)+f\left(\frac{a+\theta b}{\theta+1}\right)}{2}$ for $\theta>0$. It means that

(1) Theorem 6 is better than Theorem 2,

(2) In Theorem 6 if one takes $\theta=1$, one has (1.1),

(3) Theorem 6 is not a result of Theorem 1. 


\section{NEW FRACTIONAL MIDPOINT TYPE INEQUALITIES}

We will now prove an equality to have new fractional midpoint type inequalities.

Lemma 4. Let $a, b \in \mathbb{R}$ with $a<b$ and $f:[a, b] \rightarrow \mathbb{R}$ be a differentiable function on $(a, b)$. If $f^{\prime} \in L[a, b]$, then the following equality for the fractional integrals holds:

$$
\begin{array}{r}
\frac{\Gamma(\theta+1)}{2(b-a)^{\theta}}\left[R_{a+}^{\theta} f(b)+R_{b-}^{\theta} f(a)\right]-\frac{f\left(\frac{\theta a+b}{\theta+1}\right)+f\left(\frac{a+\theta b}{\theta+1}\right)}{2} \\
=\frac{b-a}{2}\left[\begin{array}{c}
\int_{0}^{\frac{\theta}{\theta+1}} t^{\theta} f^{\prime}(t a+(1-t) b) d t \\
+\int_{\frac{\theta}{\theta+1}}^{1}\left(t^{\theta}-1\right) f^{\prime}(t a+(1-t) b) d t \\
+\int_{0}^{\theta+1}-t^{\theta} f^{\prime}(t b+(1-t) a) d t \\
+\int_{\frac{\theta}{\theta+1}}^{1}\left(1-t^{\theta}\right) f^{\prime}(t b+(1-t) a) d t
\end{array}\right]
\end{array}
$$

Proof. If (1.7) and (1.9) gather side by side and dividing into 2, it is hold the desired result.

Corollary 1. In Lemma 4, if one takes $\theta=1$, one has Lemma 1.

Theorem 7. Let $a, b \in \mathbb{R}$ with $a<b$ and $f:[a, b] \rightarrow \mathbb{R}$ be a differentiable function on $(a, b)$. If $\left|f^{\prime}\right|$ is convex on $[a, b]$, then the following fractional midpoint type inequality holds:

$$
\begin{gathered}
\left|\frac{\Gamma(\theta+1)}{2(b-a)^{\theta}}\left[R_{a+}^{\theta} f(b)+R_{b-}^{\theta} f(a)\right]-\frac{f\left(\frac{\theta a+b}{\theta+1}\right)+f\left(\frac{a+\theta b}{\theta+1}\right)}{2}\right| \\
\leq(b-a) \frac{\theta^{\theta+1}}{(\theta+1)^{\theta+2}}\left[\left|f^{\prime}(a)\right|+\left|f^{\prime}(b)\right|\right]
\end{gathered}
$$

with $\theta>0$.

Proof. Using Lemma 4 and the convexity of $\left|f^{\prime}\right|$, we have

$$
\begin{array}{r}
\left|\frac{\Gamma(\theta+1)}{2(b-a)^{\theta}}\left[R_{a+}^{\theta} f(b)+R_{b-}^{\theta} f(a)\right]-\frac{f\left(\frac{\theta a+b}{\theta+1}\right)+f\left(\frac{a+\theta b}{\theta+1}\right)}{2}\right| \\
\frac{b-a}{2}\left[\begin{array}{c}
\int_{0}^{\frac{\theta}{\theta+1}} t^{\theta}\left|f^{\prime}(t a+(1-t) b)\right| d t \\
+\int_{\frac{\theta}{\theta+1}}^{1}\left(1-t^{\theta}\right)\left|f^{\prime}(t a+(1-t) b)\right| d t \\
+\int_{0}^{\frac{\theta}{\theta+1}} t^{\theta}\left|f^{\prime}(t b+(1-t) a)\right| d t \\
+\int_{\frac{\theta}{\theta+1}}^{1}\left(1-t^{\theta}\right)\left|f^{\prime}(t b+(1-t) a)\right| d t
\end{array}\right]
\end{array}
$$




$$
\begin{gathered}
\leq \frac{b-a}{2}\left[\begin{array}{c}
\int_{0}^{\frac{\theta}{\theta+1}} t^{\theta}\left[t\left|f^{\prime}(a)\right|+(1-t)\left|f^{\prime}(b)\right|\right] d t \\
+\int_{\frac{\theta}{\theta+1}}^{1}\left(1-t^{\theta}\right)\left[t\left|f^{\prime}(a)\right|+(1-t)\left|f^{\prime}(b)\right|\right] d t \\
+\int_{0}^{\frac{\theta}{\theta+1}} t^{\theta}\left[t\left|f^{\prime}(b)\right|+(1-t)\left|f^{\prime}(a)\right|\right] d t \\
+\int_{\frac{\theta}{\theta+1}}^{1}\left(1-t^{\theta}\right)\left[t\left|f^{\prime}(b)\right|+(1-t)\left|f^{\prime}(a)\right|\right] d t
\end{array}\right] \\
=\frac{b-a}{2}\left[\begin{array}{c}
\int_{0}^{\frac{\theta}{\theta+1}} t^{\theta}\left[\left|f^{\prime}(a)\right|+\left|f^{\prime}(b)\right|\right] d t \\
+\int_{\frac{\theta}{\theta+1}}^{1}\left(1-t^{\theta}\right)\left[\left|f^{\prime}(a)\right|+\left|f^{\prime}(b)\right|\right] d t
\end{array}\right] \\
=\frac{b-a}{2}\left[\int_{0}^{\frac{\theta}{\theta+1}} t^{\theta} d t+\int_{\frac{\theta}{\theta+1}}^{1}\left(1-t^{\theta}\right) d t\right]\left[\left|f^{\prime}(a)\right|+\left|f^{\prime}(b)\right|\right] \\
=(b-a) \frac{\theta^{\theta+1}}{(\theta+1)^{\theta+2}}\left[\left|f^{\prime}(a)\right|+\left|f^{\prime}(b)\right|\right] .
\end{gathered}
$$

This completes the proof.

Corollary 2. In Theorem 7, if one takes $\theta=1$, one has [5, Theorem 2.2].

Theorem 8. Let $a, b \in \mathbb{R}$ with $a<b$ and $f:[a, b] \rightarrow \mathbb{R}$ be a differentiable function on $(a, b)$. If $\left|f^{\prime}\right|^{q}$ is convex on $[a, b]$ for $q \geq 1$, then the following fractional midpoint type inequality holds:

$$
\begin{gathered}
\left|\frac{\Gamma(\theta+1)}{2(b-a)^{\theta}}\left[R_{a+}^{\theta} f(b)+R_{b-}^{\theta} f(a)\right]-\frac{f\left(\frac{\theta a+b}{\theta+1}\right)+f\left(\frac{a+\theta b}{\theta+1}\right)}{2}\right| \\
\leq \frac{b-a}{2} \frac{\theta^{\theta+1}}{(\theta+1)^{\theta+2}}\left[\begin{array}{c}
+\left(\frac{\theta}{\theta+2}\left|f^{\prime}(a)\right|^{q}+\frac{2}{\theta+2}\left|f^{\prime}(b)\right|^{q}\right)^{\frac{1}{q}} \\
+\left(\frac{(\theta+1)^{\theta}+2 \theta^{\theta+1}}{2 \theta^{\theta}(\theta+2)}\left|f^{\prime}(a)\right|^{q}+\frac{4 \theta^{\theta}-(\theta+1)^{\theta}}{2 \theta^{\theta}(\theta+2)}\left|f^{\prime}(b)\right|^{q}\right)^{\frac{1}{q}} \\
+\left(\frac{\theta}{\theta+2}\left|f^{\prime}(b)\right|^{q}+\frac{2}{\theta+2}\left|f^{\prime}(a)\right|^{q}\right)^{\frac{1}{q}} \\
+\left(\frac{(\theta+1)^{\theta}+2 \theta^{\theta+1}}{2 \theta^{\theta}(\theta+2)}\left|f^{\prime}(b)\right|^{q}+\frac{4 \theta^{\theta}-(\theta+1)^{\theta}}{2 \theta^{\theta}(\theta+2)}\left|f^{\prime}(a)\right|^{q}\right)^{\frac{1}{q}}
\end{array}\right]
\end{gathered}
$$

with $\theta>0$.

Proof. Using Lemma 4, power mean inequality and the convexity of $\left|f^{\prime}\right|^{q}$, we have

$$
\left|\frac{\Gamma(\theta+1)}{2(b-a)^{\theta}}\left[R_{a+}^{\theta} f(b)+R_{b-}^{\theta} f(a)\right]-\frac{f\left(\frac{\theta a+b}{\theta+1}\right)+f\left(\frac{a+\theta b}{\theta+1}\right)}{2}\right|
$$




$$
\begin{aligned}
& \leq \frac{b-a}{2}\left[\begin{array}{c}
\int_{0}^{\frac{\theta}{\theta+1}} t^{\theta}\left|f^{\prime}(t a+(1-t) b)\right| d t \\
+\int_{\frac{\theta}{\theta+1}}^{1}\left(1-t^{\theta}\right)\left|f^{\prime}(t a+(1-t) b)\right| d t \\
+\int_{0}^{\frac{\theta}{\theta+1}} t^{\theta}\left|f^{\prime}(t b+(1-t) a)\right| d t \\
+\int_{\frac{\theta}{\theta+1}}^{1}\left(1-t^{\theta}\right)\left|f^{\prime}(t b+(1-t) a)\right| d t
\end{array}\right] \\
& \leq \frac{b-a}{2}\left[\begin{array}{c}
\left(\int_{0}^{\frac{\theta}{\theta+1}} t^{\theta} d t\right)^{1-\frac{1}{q}}\left(\int_{0}^{\frac{\theta}{\theta+1}} t^{\theta}\left|f^{\prime}(t a+(1-t) b)\right|^{q} d t\right)^{\frac{1}{q}} \\
+\left(\int_{\frac{\theta}{\theta+1}}^{1}\left(1-t^{\theta}\right) d t\right)^{1-\frac{1}{q}}\left(\int_{\frac{\theta}{\theta+1}}^{1}\left(1-t^{\theta}\right)\left|f^{\prime}(t a+(1-t) b)\right|^{q} d t\right)^{\frac{1}{q}} \\
+\left(\int_{0}^{\frac{\theta}{\theta+1}} t^{\theta} d t\right)^{1-\frac{1}{q}}\left(\int_{0}^{\frac{\theta}{\theta+1}} t^{\theta}\left|f^{\prime}(t b+(1-t) a)\right|^{q} d t\right)^{\frac{1}{q}} \\
+\left(\int_{\frac{\theta}{\theta+1}}^{1}\left(1-t^{\theta}\right) d t\right)^{1-\frac{1}{q}}\left(\int_{\frac{\theta}{\theta+1}}^{1}\left(1-t^{\theta}\right)\left|f^{\prime}(t b+(1-t) a)\right|^{q} d t\right)^{\frac{1}{q}}
\end{array}\right] \\
& \leq \frac{b-a}{2}\left(\frac{\theta^{\theta+1}}{(\theta+1)^{\theta+2}}\right)^{1-\frac{1}{q}}\left[\begin{array}{c}
\left(\int_{0}^{\frac{\theta}{\theta+1}} t^{\theta}\left[t\left|f^{\prime}(a)\right|^{q}+(1-t)\left|f^{\prime}(b)\right|^{q}\right] d t\right)^{\frac{1}{q}} \\
+\left(\int_{\frac{\theta}{\theta+1}}^{1}\left(1-t^{\theta}\right)\left[t\left|f^{\prime}(a)\right|^{q}+(1-t)\left|f^{\prime}(b)\right|^{q}\right] d t\right)^{\frac{1}{q}} \\
+\left(\int_{0}^{\frac{\theta}{\theta+1}} t^{\theta}\left[t\left|f^{\prime}(b)\right|^{q}+(1-t)\left|f^{\prime}(a)\right|^{q}\right] d t\right)^{\frac{1}{q}} \\
+\left(\int_{\frac{\theta}{\theta+1}}^{1}\left(1-t^{\theta}\right)\left[t\left|f^{\prime}(b)\right|^{q}+(1-t)\left|f^{\prime}(a)\right|^{q}\right] d t\right)^{\frac{1}{q}}
\end{array}\right] \\
& \leq \frac{b-a}{2} \frac{\theta^{\theta+1}}{(\theta+1)^{\theta+2}}\left[\begin{array}{c}
\left(\frac{\theta}{\theta+2}\left|f^{\prime}(a)\right|^{q}+\frac{2}{\theta+2}\left|f^{\prime}(b)\right|^{q}\right)^{\frac{1}{q}} \\
+\left(\frac{(\theta+1)^{\theta}+2 \theta^{\theta+1}}{2 \theta^{\theta}(\theta+2)}\left|f^{\prime}(a)\right|^{q}+\frac{4 \theta^{\theta}-(\theta+1)^{\theta}}{2 \theta^{\theta}(\theta+2)}\left|f^{\prime}(b)\right|^{q}\right)^{\frac{1}{q}} \\
+\left(\frac{\theta}{\theta+2}\left|f^{\prime}(b)\right|^{q}+\frac{2}{\theta+2}\left|f^{\prime}(a)\right|^{q}\right)^{\frac{1}{q}} \\
+\left(\frac{(\theta+1)^{\theta}+2 \theta^{\theta+1}}{2 \theta^{\theta}(\theta+2)}\left|f^{\prime}(b)\right|^{q}+\frac{4 \theta^{\theta}-(\theta+1)^{\theta}}{2 \theta^{\theta}(\theta+2)}\left|f^{\prime}(a)\right|^{q}\right)^{\frac{1}{q}}
\end{array}\right] .
\end{aligned}
$$

This completes the proof.

Corollary 3. In Theorem 8 , if one takes $\theta=1$, one has the following midpoint type inequality,

$$
\left|\frac{1}{b-a} \int_{a}^{b} f(u) d u-f\left(\frac{a+b}{2}\right)\right| \leq \frac{b-a}{8}\left[\begin{array}{c}
\left(\frac{1}{3}\left|f^{\prime}(a)\right|^{q}+\frac{2}{3}\left|f^{\prime}(b)\right|^{q}\right)^{\frac{1}{q}} \\
+\left(\frac{2}{3}\left|f^{\prime}(a)\right|^{q}+\frac{1}{3}\left|f^{\prime}(b)\right|^{q}\right)^{\frac{1}{q}}
\end{array}\right]
$$

Theorem 9. Let $a, b \in \mathbb{R}$ with $a<b$ and $f:[a, b] \rightarrow \mathbb{R}$ be a differentiable function on $(a, b)$. If $\left|f^{\prime}\right|^{q}$ is convex on $[a, b]$ for $q>1$, then the following fractional midpoint 
type inequality holds:

$$
\begin{aligned}
& \left|\frac{\Gamma(\theta+1)}{2(b-a)^{\theta}}\left[R_{a+}^{\theta} f(b)+R_{b-}^{\theta} f(a)\right]-\frac{f\left(\frac{\theta a+b}{\theta+1}\right)+f\left(\frac{a+\theta b}{\theta+1}\right)}{2}\right| \\
& \leq \frac{b-a}{2}\left[\begin{array}{c}
\left(\int_{0}^{\frac{\theta}{\theta+1}} t^{\theta p} d t\right)^{\frac{1}{p}}\left(\frac{\theta^{2}}{2(\theta+1)^{2}}\left|f^{\prime}(a)\right|^{q}+\frac{\theta^{2}+2 \theta}{2(\theta+1)^{2}}\left|f^{\prime}(b)\right|^{q}\right)^{\frac{1}{q}} \\
+\left(\int_{\frac{\theta}{\theta+1}}^{1}\left(1-t^{\theta}\right)^{p} d t\right)^{\frac{1}{p}}\left(\frac{2 \theta+1}{2(\theta+1)^{2}}\left|f^{\prime}(a)\right|^{q}+\frac{1}{2(\theta+1)^{2}}\left|f^{\prime}(b)\right|^{q}\right)^{\frac{1}{q}} \\
+\left(\int_{0}^{\frac{\theta}{\theta+1}} t^{\theta p} d t\right)^{\frac{1}{p}}\left(\frac{\theta^{2}}{2(\theta+1)^{2}}\left|f^{\prime}(b)\right|^{q}+\frac{\theta^{2}+2 \theta}{2(\theta+1)^{2}}\left|f^{\prime}(a)\right|^{q}\right)^{\frac{1}{q}} \\
+\left(\int_{\frac{\theta}{\theta+1}}^{1}\left(1-t^{\theta}\right)^{p} d t\right)^{\frac{1}{p}}\left(\frac{2 \theta+1}{2(\theta+1)^{2}}\left|f^{\prime}(b)\right|^{q}+\frac{1}{2(\theta+1)^{2}}\left|f^{\prime}(a)\right|^{q}\right)^{\frac{1}{q}}
\end{array}\right]
\end{aligned}
$$

with $\frac{1}{p}+\frac{1}{q}=1$ and $\theta>0$.

Proof. Using Lemma 4, Holder inequality and the convexity of $\left|f^{\prime}\right|^{q}$, we have

$$
\begin{aligned}
& \left|\frac{\Gamma(\theta+1)}{2(b-a)^{\theta}}\left[R_{a+}^{\theta} f(b)+R_{b-}^{\theta} f(a)\right]-\frac{f\left(\frac{\theta a+b}{\theta+1}\right)+f\left(\frac{a+\theta b}{\theta+1}\right)}{2}\right| \\
& \leq \frac{b-a}{2}\left[\begin{array}{c}
\int_{0}^{\frac{\theta}{\theta+1}} t^{\theta}\left|f^{\prime}(t a+(1-t) b)\right| d t \\
+\int_{\frac{\theta}{\theta+1}}^{1}\left(1-t^{\theta}\right)\left|f^{\prime}(t a+(1-t) b)\right| d t \\
+\int_{0}^{\theta+1} t^{\theta}\left|f^{\prime}(t b+(1-t) a)\right| d t \\
+\int_{\frac{\theta}{\theta+1}}^{1}\left(1-t^{\theta}\right)\left|f^{\prime}(t b+(1-t) a)\right| d t
\end{array}\right] \\
& \leq \frac{b-a}{2}\left[\begin{array}{c}
\left(\int_{0}^{\frac{\theta}{\theta+1}} t^{\theta p} d t\right)^{\frac{1}{p}}\left(\int_{0}^{\frac{\theta}{\theta+1}}\left|f^{\prime}(t a+(1-t) b)\right|^{q} d t\right)^{\frac{1}{q}} \\
+\left(\int_{\frac{\theta}{\theta+1}}^{1}\left(1-t^{\theta}\right)^{p} d t\right)^{\frac{1}{p}}\left(\int_{\frac{\theta}{\theta+1}}^{1}\left|f^{\prime}(t a+(1-t) b)\right|^{q} d t\right)^{\frac{1}{q}} \\
+\left(\int_{0}^{\frac{\theta}{\theta+1}} t^{\theta p} d t\right)^{\frac{1}{p}}\left(\int_{0}^{\frac{\theta}{\theta+1}}\left|f^{\prime}(t b+(1-t) a)\right|^{q} d t\right)^{\frac{1}{q}} \\
+\left(\int_{\frac{\theta}{\theta+1}}^{1}\left(1-t^{\theta}\right)^{p} d t\right)^{\frac{1}{p}}\left(\int_{\frac{\theta}{\theta+1}}^{1}\left|f^{\prime}(t b+(1-t) a)\right|^{q} d t\right)^{\frac{1}{q}}
\end{array}\right] \\
& {\left[\left(\int_{0}^{\frac{\theta}{\theta+1}} t^{\theta p} d t\right)^{\frac{1}{p}}\left(\int_{0}^{\frac{\theta}{\theta+1}}\left[t\left|f^{\prime}(a)\right|^{q}+(1-t)\left|f^{\prime}(b)\right|^{q}\right] d t\right)^{\frac{1}{q}}\right.} \\
& \leq \frac{b-a}{2}+\left(\int_{\frac{\theta}{\theta+1}}^{1}\left(1-t^{\theta}\right)^{p} d t\right)^{\frac{1}{p}}\left(\int_{\frac{\theta}{\theta+1}}^{1}\left[t\left|f^{\prime}(a)\right|^{q}+(1-t)\left|f^{\prime}(b)\right|^{q}\right] d t\right)^{\frac{1}{q}} \\
& +\left(\int_{0}^{\frac{\theta}{\theta+1}} t^{\theta p} d t\right)^{\frac{1}{p}}\left(\int_{0}^{\frac{\theta}{\theta+1}}\left[t\left|f^{\prime}(b)\right|^{q}+(1-t)\left|f^{\prime}(a)\right|^{q}\right] d t\right)^{\frac{1}{q}} \\
& \left.+\left(\int_{\frac{\theta}{\theta+1}}^{1}\left(1-t^{\theta}\right)^{p} d t\right)^{\frac{1}{p}}\left(\int_{\frac{\theta}{\theta+1}}^{1}\left[t\left|f^{\prime}(b)\right|^{q}+(1-t)\left|f^{\prime}(a)\right|^{q}\right] d t\right)^{\frac{1}{q}}\right]
\end{aligned}
$$




$$
\leq \frac{b-a}{2}\left[\begin{array}{c}
\left(\int_{0}^{\frac{\theta}{\theta+1}} t^{\theta p} d t\right)^{\frac{1}{p}}\left(\frac{\theta^{2}}{2(\theta+1)^{2}}\left|f^{\prime}(a)\right|^{q}+\frac{\theta^{2}+2 \theta}{2(\theta+1)^{2}}\left|f^{\prime}(b)\right|^{q}\right)^{\frac{1}{q}} \\
+\left(\int_{\frac{\theta}{\theta+1}}^{1}\left(1-t^{\theta}\right)^{p} d t\right)^{\frac{1}{p}}\left(\frac{2 \theta+1}{2(\theta+1)^{2}}\left|f^{\prime}(a)\right|^{q}+\frac{1}{2(\theta+1)^{2}}\left|f^{\prime}(b)\right|^{q}\right)^{\frac{1}{q}} \\
+\left(\int_{0}^{\frac{\theta}{\theta+1}} t^{\theta p} d t\right)^{\frac{1}{p}}\left(\frac{\theta^{2}}{2(\theta+1)^{2}}\left|f^{\prime}(b)\right|^{q}+\frac{\theta^{2}+2 \theta}{2(\theta+1)^{2}}\left|f^{\prime}(a)\right|^{q}\right)^{\frac{1}{q}} \\
+\left(\int_{\frac{\theta}{\theta+1}}^{1}\left(1-t^{\theta}\right)^{p} d t\right)^{\frac{1}{p}}\left(\frac{2 \theta+1}{2(\theta+1)^{2}}\left|f^{\prime}(b)\right|^{q}+\frac{1}{2(\theta+1)^{2}}\left|f^{\prime}(a)\right|^{q}\right)^{\frac{1}{q}}
\end{array}\right] .
$$

This completes the proof.

Corollary 4. In Theorem 9, if one takes $\theta=1$, one has [5, Theorem 2.3].

\section{COMPETING INTERESTS}

The authors declare that they have no competing interests.

\section{REFERENCES}

[1] L. Fejér, "Uber die Fourierreihen, II," Math. Naturwise. Anz. Ungar. Akad., Wiss, no. 24, pp. 369-390, 1906.

[2] J. Hadamard, "Étude sur les propriétés des fonctions entières et en particulier d'une fonction considérée par Riemann," J. Math. Pures Appl., no. 58, pp. 171-215, 1893.

[3] C. Hermite, "Sur deux limites d'une intégrale définie," Mathesis, no. 3, pp. 82-83, 1883.

[4] I. İşcan, "Hermite-Hadamard-Fejer type inequalities for convex functions via fractional integrals," Stud. Univ. Babeş-Bolyai Math., vol. 60, no. 3, pp. 355-366, 2015.

[5] U. S. Kırmac1, "Inequalities for differentiable mappings and applications to special means of real numbers and to midpoint formula," Appl. Math. Comp., no. 147, pp. 137-146, 2004.

[6] A. A. Kilbas, H. M. Srivastava, and J. J. Trujillo, Theory and applications of fractional differential equations. Amsterdam: Elsevier, 2006.

[7] M. Kunt, D. Karapınar, S. Turhan, and I. İşcan, "The left Rieaman-Liouville fractional HermiteHadamard type inequalities for convex functions," RGMIA Res. Rep. Coll., vol. 20, no. Article:101, pp. 1-8, 2017.

[8] M. Kunt, D. Karapınar, S. Turhan, and I. İşcan, "The right Rieaman-Liouville fractional HermiteHadamard type inequalities for convex functions," RGMIA Res. Rep. Coll., vol. 20, no. Article:102, pp. 1-8, 2017.

[9] M. Sarıkaya, E. Set, H. Yaldız, and N. Başak, "Hermite-Hadamard's inequalities for fractional integrals and related fractional inequalities," Math. Computer Model., vol. 57, no. 9, pp. 2403 2407, 2013.

[10] Y. Zhou, Basic theory of fractional differential equations. New Jersey: World Scientific, 2014.

Authors' addresses

\section{Mehmet Kunt}

Karadeniz Technical University, Department of Mathematics, 61080 Trabzon, Turkey

E-mail address: mkunt@ktu.edu.tr

İmdat İşcan

Giresun University, Department of Mathematics, 28200 Giresun, Turkey

E-mail address: imdati@yahoo.com; imdat. iscan@giresun.edu.tr 
Sercan Turhan

Giresun University, Department of Mathematics, 28200 Giresun, Turkey

E-mail address: sercan.turhan@giresun.edu.tr

\section{Dünya Karapınar}

Karadeniz Technical University, Department of Mathematics, 61080 Trabzon, Turkey

E-mail address: dunyakarapinar@ktu.edu.tr 